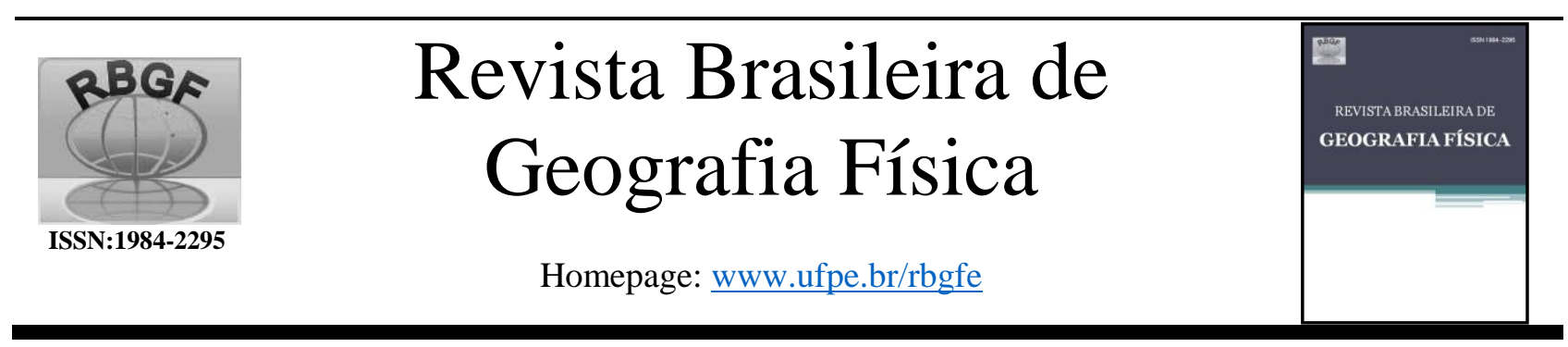

\title{
Proposta de um Roteiro Geral para Elaboração e Verificação da Qualidade do Estudo de Impacto Ambiental (EIA)
}

\author{
Ana Luisa Guimarães Ribeiro, Universidade Federal de Uberlândia. E-mail: anagr104@ gmail.com (autor \\ correspondente). Maria Rita Raimundo e Almeida. Universidade Federal de Itajubá, e-mail: \\ maria.rita.ralmeida@gmail.com
}

Artigo recebido em 11/05/2018 e aceito em 14/12/2018

\begin{abstract}
R E S U M O
A Avaliação de Impacto Ambiental é considerada de grande importância no processo decisório para licenciamento de empreendimentos capazes de causar significativos impactos ambientais. Além disso, em projetos de grande porte e alto potencial poluidor é necessário um estudo, conhecido como Estudo de Impacto Ambiental (EIA), para uma melhor e mais aprofundada análise dos impactos e das áreas atingidas, tanto nos aspectos físico e biótico como antrópico. Contudo, ainda são elaborados EIAs de má qualidade, que possuem alta subjetividade e ausência de critérios para as avaliações realizadas. Isto faz com que o EIA não cumpra seu objetivo e, ainda, dificulta e retarda a tomada de decisão. Já existem guias de elaboração e análise de EIAs, porém este trabalho propõe um roteiro geral, em português, que pode ser aplicado a qualquer tipologia de empreendimento e que irá auxiliar a elaboração e/ou a avaliação desse estudo. Para tal elaboração foram utilizadas as informações de diversos guias obtidos por meio de um levantamento de trabalhos com as mais importantes e utilizadas listas de verificação disponíveis mundialmente. Com a lista já criada e definida, espera-se que ela possa contribuir para que os estudos de maior qualidade sejam elaborados e, com isso, haja uma melhora na aplicação dos instrumentos Avaliação de Impacto e Licenciamento Ambiental.

Palavras-chave: Avaliação de Impacto Ambiental, Estudo de Impacto Ambiental, Checklist, Lista de Verificação.
\end{abstract}

\section{Proposal for a General Report on the Elaboration and Verification of the Quality of the Environmental Impact Study (EIA)}

\begin{abstract}
A B S T R A C T
The Environmental Impact Assessment is considered of great importance in the decision making process for the licensing of projects capable of causing significant environmental impacts. In addition, in large-scale projects with high polluting potential, a study, known as the Environmental Impact Study (EIA), is needed for a better and more in-depth analysis of impacts and affected areas, both in physical, biotic and anthropic aspects. However, EIAs of poor quality are still elaborated, which have high subjectivity and lack of criteria for the evaluations carried out. This means that the EIA does not fulfill its purpose and further complicates and delays decision-making. There are already guides for the elaboration and analysis of EIAs, but this work proposes a general road map in Portuguese that can be applied to any type of enterprise and that will help the elaboration and / or evaluation of this study. For this elaboration the information of several guides obtained by means of a survey of works with the most important and used lists of checking was used worldwide. With the list already created and defined, it is hoped that it can contribute to the higher quality studies being elaborated and, with this, there will be an improvement in the application of the Impact Assessment and Environmental Licensing instruments.

keywords: Environmental Impact Assessment, Environmental Impact Study, Checklist, Verification List.
\end{abstract}

\section{Introdução}

A consciência ambiental e a proteção dos recursos naturais ganharam espaço a partir das décadas de 1960 e 1970 quando passaram a integrar os princípios do homem moderno. Tais princípios fazem com que este não se preocupe apenas com o controle e mitigação de seus impactos, como também com o desempenho ambiental (Cremonez et al., 2014). Como resposta às pressões crescentes da sociedade organizada para que os aspectos ambientais passassem a ser considerados na tomada de decisão sobre a implantação de projetos capazes de causar significativa degradação ambiental (Sandoval e Cerri, 2009), entrou em vigor no dia $1^{\circ}$ de janeiro de 1970 a lei da política nacional do meio ambiente nos Estados Unidos, o 
National Environmental Policy Act (NEPA), em que instituiu Avaliação de Impacto Ambiental (AIA). Esta lei representou um marco mundial na questão ambiental, pois serviu como exemplo a ser seguido pelos outros países.

A AIA compreende o processo de identificar, prever, avaliar e mitigar os efeitos relevantes de natureza biofísica, social e outros efeitos de atividades ou projetos de desenvolvimento, antes que decisões importantes sejam tomadas (IAIA, 1999). Pode ainda ser vista como um processo de exame e de negociação do conjunto das consequências de um projeto (Leduc e Raymond, 2000), foi incorporada em processos de tomada de decisão sobre empreendimentos potencialmente poluidores e permitiu a negociação com vistas à redução de impactos ambientais (Morgan, 2012).

Um ponto fundamental quanto às suas origens é que o instrumento não nasceu pronto, mas como uma ideia a ser desenvolvida. Assim, resultou de um processo político que buscou atender a uma demanda social, que estava mais madura nos Estados Unidos no final da década 1960; por outro lado, a AIA evoluiu ao longo do tempo e foi modificada conforme lições eram aprendidas na experiência prática (Sánchez, 2013). A incorporação do planejamento estratégico e a dissolução de políticas públicas ambientais, através de programas de controle de danos ambientais, surgiram como mecanismos de contenção de impactos devido à expansão humana e a sociedade desenvolvimentista. Assim, o crescimento populacional aliado às premissas do capitalismo, através das atividades industriais, são responsáveis pelas maiores modificações ambientais até os dias de hoje (Junior, Alvarenga e Garcia, 2018).

A AIA, como instrumento de decisão na aprovação de projetos, atividades e mesmo políticas, leis, planos e programas, encontra-se hoje disseminada amplamente em todo o mundo, seja inserida no sistema de proteção do meio ambiente de muitos países, na forma de leis ou procedimentos administrativos, seja pela atuação de organismos internacionais, dos quais dependem muito dos grandes projetos de infraestrutura dos países em desenvolvimento (Sandoval e Cerri, 2009).

No Brasil, a AIA aportou na legislação federal no início dos anos oitenta, tendo como principal marco de introdução a Lei Federal nº 6938, de 31 agosto de 1981, que instituiu a Política Nacional do Meio Ambiente - PNMA (Sandoval e Cerri, 2009), sendo a AIA definida como instrumento de gestão ambiental, assim como o licenciamento ambiental. No entanto, somente com a Resolução CONAMA (Conselho Nacional do Meio Ambiente) $n^{\circ} 001$, de 1986 é que foram propostos os critérios básicos e as diretrizes gerais para a Avaliação de Impacto Ambiental. Nesta resolução ganha importância a figura do Estudo de Impacto Ambiental (EIA), sendo também estabelecidas as suas diretrizes gerais e suas atividades técnicas mínimas (Conama, 1986).

O EIA é um conjunto de procedimentos, alguns de natureza técnico-científicos, outros de natureza administrativa, destinados, primeiramente, a fazer com que os impactos ambientais de um projeto sejam sistematicamente analisados e, em segundo lugar, que assegurem os resultados da análise, controlando efeitos ambientais esperados (Tommasi, 1994). Complementando, como um dos elementos do processo de AIA, o EIA trata-se do estudo detalhado (técnico multidisciplinar) no qual se identificam e se valoram os impactos ambientais de um projeto ou de um empreendimento (Almeida et al., 2012). Espera-se que a visão multidisciplinar faça com que o estudo seja feito da forma mais completa possível, de modo a sanar todas as dúvidas e problemas (Leite, 2013). Em termos de conteúdo, o EIA deve apresentar uma síntese clara e concisa da caracterização do empreendimento, da condição do meio onde será implantado o projeto (diagnóstico ambiental), descrever os prováveis impactos ambientais (prognóstico ambiental), caracterizar as propostas de medidas de mitigação e compensatórias, a importância dos impactos residuais e apresentar sugestões para outros estudos de acompanhamento (Ross, Morrison-Saunders e Marshall, 2006). Já o Relatório de Impacto Ambiental (RIMA) é exigido nos mesmos casos em que se exige o EIA e reflete todas as conclusões apresentadas neste Estudo. Deve ser elaborado de forma objetiva e de fácil compreensão por parte da população. Se for solicitado, deve respeitar o sigilo industrial (Leite, 2013).

Assim, o EIA pode ser entendido como parte central do processo de AIA. No entanto, a literatura aponta que os estudos ambientais têm baixa qualidade e apresentam deficiências de conteúdo: proposição de alternativas tecnológicas e locacionais incipiente ou inexistente (Pinho, Maia e Monterroso, 2007; Kruopiene, Židoniene e Dvarioniene, 2009; Pope et al., 2013) ou que abrange alternativas ambientalmente inviáveis (Zubair, 2001); inadequada definição da área de influência; diagnósticos superficiais e incompletos (Gomes et al., 2009; Panigrahi e Amirapu, 2012); bases inadequadas de dados (Hickie e Wade, 1998; 
Zubair, 2001); inapropriada identificação, previsão e avaliação de impactos (Appiah-Opoku, 2001; Panigrahi e Amirapu, 2012); tratamento insuficiente de impactos cumulativos (Zubair, 2001; Wärnbäc e Hilding-Rydevika, 2009; Pope et al., 2013); proposição de medidas que não são a solução para o impacto (Prado Filho e Souza, 2004); os procedimentos aplicados nos diferentes entes federativos mostram lacunas legais e técnicas, defasando as premissas do planejamento sustentável (Junior, Alvarenga e Garcia, 2018); e Tzoumis (2007) expõe sobre as deficiências dos estudos ambientais e desqualificação do corpo técnico incumbido de analisar os estudos. Da mesma forma, pesquisadores como Almeida, Alvarenga e Céspedes (2014) e Santiago, Alvarenga e Almeida (2016) também criticam a efetividade do EIA nos processos de licenciamento, solicitação de estudos ambientais incapazes de fornecer informações que atestem a viabilidade ambiental dos empreendimentos, inconformidade legal e falta de capacidade técnica dos órgãos competentes. Sendo assim, torna-se irrefutável a adesão de ferramentas de aperfeiçoamento no panorama normativo e técnico-executivo (Junior, Alvarenga e Garcia, 2018).

Bem voltados para a realidade brasileira, os trabalhos de MPF (2004) e Lima e Magrini (2010) também apontam para estudos ambientais de baixa qualidade. O primeiro fez um levantamento onde foram identificadas as principais deficiências que se relacionam com a elaboração dos estudos ambientais; e o segundo apresenta o resultado de uma auditoria do Tribunal de Contas da União (TCU) que entre as suas principais conclusões apontaram deficiências nos estudos ambientais componentes do processo de licenciamento. A situação agrava-se porque para um mesmo local podem coexistir projetos licenciados por diferentes esferas governamentais, cuja integração é precária (Turra et al., 2017).

Esta má qualidade dos estudos ambientais é apontada como um dos principais problemas da aplicação da AIA e do licenciamento ambiental
(Mendes e Feitosa, 2010), pelo fato de não fornecerem as informações necessárias para uma boa decisão (Tzoumis, 2007). Na questão da AIA em si é relevante mencionar que ela não se refere somente a um mecanismo de mitigação de impactos ambientais, sua expansão reflete uma integração ao desenvolvimento de projetos, permitindo satisfatório desempenho social (Vanclay, 2015).

Uma das razões para haver tanta problemática na qualidade dos EIAs pode ser estar na falta de suporte para sua elaboração: os termos de referência disponibilizados pelos órgãos ambientais são gerais e focam em conteúdo e não em orientações para a sua elaboração.

Atualmente, já existem roteiros para elaboração e avaliação da qualidade dos EIAs, sendo sua grande maioria disponível na língua inglesa. Já no Brasil, o mais utilizado é o guia desenvolvido por Sanchéz (2008), contudo o mesmo foi elaborado para rodovias e é necessária sua adaptação para outras tipologias de empreendimento.

Dessa forma, este trabalho objetiva elaborar um roteiro geral, em português, que oriente a elaboração e/ou a análise técnica do Estudo de Impacto Ambiental de qualquer tipologia de empreendimentos.

\section{Material e métodos}

A metodologia do trabalho consistiu em três etapas, sendo elas: levantamento de dados, definição de critérios essenciais e elaboração do roteiro/lista de verificação.

A primeira etapa, levantamento de dados, consistiu em encontrar as listas de verificação usualmente utilizadas mundialmente. Este levantamento baseou-se, inicialmente, em um estudo do livro de Sánchez (2013) e de suas recomendações em roteiros reconhecidos e utilizados em trabalhos renomados, o quadro de citações pode ser conferido na Tabela 1. A partir destes trabalhos, outros foram levantados e assim sucessivamente até que foram selecionados os principais métodos utilizados como base para a elaboração e revisão de EIA.

Tabela 1. Síntese de estudos sobre a qualidade de EIAs. Fonte: Sánchez (2013).

\begin{tabular}{|c|c|c|c|c|}
\hline Autores & Amostra & Local & Período & Métodos \\
\hline $\begin{array}{l}\text { Lee e Brown } \\
\quad(1992)\end{array}$ & $\begin{array}{c}83 \text { EIAs / } \\
\text { Vários setores }\end{array}$ & Reino Unido & & Procedimento de \\
\hline $\begin{array}{l}\text { Lee e Dancey } \\
\quad(1993)\end{array}$ & $\begin{array}{c}40 \text { EIAs / } \\
\text { Vários setores }\end{array}$ & Irlanda & 1988-1992 & análise de Lee e Colley (1999) \\
\hline
\end{tabular}




\begin{tabular}{|c|c|c|c|c|}
\hline $\begin{array}{l}\text { Glasson, Therivel e } \\
\text { Chadwick (1999) }\end{array}$ & $\begin{array}{l}50 \text { EIAs / } \\
\text { Vários setores }\end{array}$ & Reino Unido & $1988-1996$ & $\begin{array}{l}\text { Comparação de pares de EIAs - por tipo } \\
\text { de projeto, localização e outras } \\
\text { características - em dois períodos }\end{array}$ \\
\hline $\begin{array}{l}\text { Bojórquez-Tapia e } \\
\text { Garcia (1998) }\end{array}$ & $\begin{array}{l}33 \text { EIAs / } \\
\text { Vários setores }\end{array}$ & México & 1989-1994 & $\begin{array}{l}\text { Conjunto de critérios próprios } \\
\text { resultando em uma nota para cada EIA }\end{array}$ \\
\hline Wende (2002) & $\begin{array}{l}145 \text { EIAs / } \\
\text { Vários setores }\end{array}$ & Alemanha & 1990-1997 & $\begin{array}{l}\text { Análise estatística } \\
\text { de } 11 \text { variáveis }\end{array}$ \\
\hline $\begin{array}{l}\text { Cashmore et al. } \\
\text { (2002) }\end{array}$ & $\begin{array}{l}72 \text { EIAs / } \\
\text { Vários setores }\end{array}$ & Grécia & 1990-1999 & Procedimento de \\
\hline $\begin{array}{l}\text { Gray e Edwards- } \\
\text { Jones }(2003\end{array}$ & $\begin{array}{l}89 \text { EIAs / } \\
\text { Setor florestal }\end{array}$ & Reino Unido & $1988-1998$ & análise de Lee e Colley (1999) \\
\hline $\begin{array}{l}\text { Canelas et al. } \\
\qquad(2005)\end{array}$ & $\begin{array}{l}46 \text { EIAs / } \\
\text { Vários setores }\end{array}$ & $\begin{array}{l}\text { Espanha e } \\
\text { Portugal }\end{array}$ & $1998-2003$ & Guia da Comissão Europeia \\
\hline $\begin{array}{l}\text { Carrasco, Blank e } \\
\quad \text { Sills (2006) }\end{array}$ & $\begin{array}{l}46 \text { EIAs / } \\
\text { Rodovias }\end{array}$ & Estados Unidos & 1980-1999 & $\begin{array}{l}\text { Obtenção de um "índice de completude" } \\
\text { para medir a quantidade de informação }\end{array}$ \\
\hline $\begin{array}{l}\text { Gontier et al. } \\
\quad(2006)\end{array}$ & $\begin{array}{l}38 \text { EIAs / } \\
\text { Vários setores }\end{array}$ & $\begin{array}{l}\text { Suécia, França, } \\
\text { Reino Unido e } \\
\text { Irlanda }\end{array}$ & $1999-2033$ & $\begin{array}{l}\text { Análise de conteúdo } \\
\text { relativo à biodiversidade }\end{array}$ \\
\hline $\begin{array}{c}\text { Pinho, Maia e } \\
\text { Monterroso (2007) }\end{array}$ & $\begin{array}{l}13 \text { EIAs / Pequenos } \\
\text { Projetos } \\
\text { Hidrelétricos }\end{array}$ & Portugal & $1990-2003$ & $\begin{array}{l}\text { Conjunto de } 12 \text { critérios e } 43 \\
\text { subcritérios } \\
\text { resultando em uma nota para cada } \\
\text { documento }\end{array}$ \\
\hline $\begin{array}{c}\text { Sandham e } \\
\text { Pretorius (2008) }\end{array}$ & $\begin{array}{l}28 \text { EIAs / } \\
\text { Vários setores }\end{array}$ & África do Sul & $\begin{array}{l}\text { Não- } \\
\text { especificado }\end{array}$ & $\begin{array}{l}\text { Versão adaptada do procedimento } \\
\text { de análise de Lee e Colley (1999) }\end{array}$ \\
\hline Javala et al. (2010). & $\begin{array}{l}15 \text { EIAs / } \\
\text { Incineradores }\end{array}$ & Finlândia & $\begin{array}{l}\text { Não- } \\
\text { especificado }\end{array}$ & $\begin{array}{l}\text { Comparação de notas atribuídas por } \\
\text { consultores e funcionários públicos, } \\
\text { segundo o Guia da Comissão Europeia }\end{array}$ \\
\hline $\begin{array}{l}\text { Kabir e Momtaz } \\
\text { (2012) }\end{array}$ & $\begin{array}{l}30 \text { EIAs / } \\
\text { Vários setores }\end{array}$ & Bangladesh & $\begin{array}{l}\text { Não- } \\
\text { especificado }\end{array}$ & $\begin{array}{l}\text { Procedimento de } \\
\text { análise de Lee e Colley (1999) }\end{array}$ \\
\hline $\begin{array}{l}\text { Landim e Sánchez } \\
\qquad \text { (2012) }\end{array}$ & $\begin{array}{l}9 \text { EIAs / } \\
\text { Mineração }\end{array}$ & Brasil & $1987-2010$ & Análise de conteúdo \\
\hline $\begin{array}{l}\text { Sandham et al. } \\
\qquad(2013)\end{array}$ & $\begin{array}{l}26 \text { EIAs / } \\
\text { Vários setores }\end{array}$ & África do Sul & $1997-2008$ & $\begin{array}{l}\text { Versão adaptada do procedimento } \\
\text { de análise de Lee e Colley (1999) }\end{array}$ \\
\hline
\end{tabular}

Assim, foram levantados métodos mundialmente reconhecidos tanto de elaboração quanto de avaliação de qualidade de estudos de impacto por listas de verificação. Os métodos/listas de verificação escolhidos para serem utilizados neste trabalho e suas justificativas de seleção são listados a seguir:

$\checkmark$ Lista de verificação de Hickie e Wade (1998): também produzida no Reino Unido e sendo mundialmente reconhecida foi recomendada para análise pela Doutora Maria Rita Raimundo e Almeida. Apesar de não se ter obtido a lista de verificação completa, os títulos principais de cada tópico da listagem estavam disponíveis o que auxiliou na elaboração do roteiro geral.

$\checkmark$ Lista de Lee e Colley (1999): extremamente citada em múltiplos trabalhos e pesquisas (conforme Tabela 1) como referência de base em montagens de listas de verificação e também para análise de estudos ambientais;
Lista de verificação de Glasson, Therivel e Chadwick (1999): produzida na Universidade de Oxford no Reino Unido, é uma lista reconhecida mundialmente e integra vários fatores de forma coerente e concisa, deixando claros os pontos principais a serem adotados em um EIA de boa qualidade;

$\checkmark$ Guia da Comissão Europeia (2001): escolhida entre os diversos guias por poder acrescentar na elaboração da lista um padrão europeu; também é altamente reconhecida e utilizada como base de referência de vários trabalhos (conforme Tabela 1);

$\checkmark$ Lista de verificação de Montaño et al. (2014) e lista de verificação de Pinho, Maia e Monterroso (2007): apesar de não estarem na Tabela 1 e serem listas de verificação voltadas para pequenas centrais hidrelétricas, utilizam como referencial outras listas de verificação (como as citadas anteriormente); 
Lista de verificação de Sánchez (2008): bastante conhecida e utilizada no Brasil, é uma lista de verificação voltada para a análise do Estudo de Impacto Ambiental de rodovias, mas que abrange todos os tópicos de um estudo completo e bem elaborado;

Para a segunda etapa, definição de critérios essenciais, analisou-se quais parâmetros devem estar contidos em roteiros de elaboração e análise de EIAs de forma a explorar todas as áreas fundamentais de um estudo de boa qualidade, abrangendo todos os temas de análise. Dessa forma, para se estabelecer estes critérios, primeiramente, foi realizada a tradução das listas selecionadas como base de elaboração do roteiro aqui proposto e, após isso, verificou-se os tópicos e subtópicos principais que todas estas listas continham. Um exemplo de como isso foi realizado está exposto na Figura 1. Da análise realizada, foi, então, selecionado para ser um dos tópicos do roteiro a caracterização do projeto. Para compor sues subtópicos foi efetuada uam composição do que as listas de referência apresentavam.

A terceira etapa, elaboração do roteiro geral/lista de verificação, fundamentou-se em utilizar das informações obtidas na primeira e na segunda etapas para elaborar um guia que pudesse ser seguido na elaboração e também na análise técnica do EIA, ao mesmo tempo em que a aplicabilidade de cada tópico deste roteiro poderá ser avaliada de acordo com as especificidades de cada caso.

Figura 1. Exemplo da seleção de um critério essencial para elaboração do guia/ lista de verificação.

\author{
LISTA SÁNCHEZ \\ 2. INFORMACŌES GERAIS \\ 2.40bjetivos do empreendimento e sua justificativa \\ 2.4.1 Objetivos são claramente expostos? \\ LISTA LEE E COLLEY \\ 1. DESCRIÇÄO DO DESENVOLVIMENTO, DO AMBIENTE LOCAL E DAS CONDICŌES DE \\ BASE \\ 1.1 Descriçăo do desenvolvimento: $O$ propósito do desenvolvimento deve ser descrito \\ de acordo com as caracteristicas físicas, escala e desing. Quantidade de materiais \\ necessários durante a construçăo $e$ operaçăo devem ser incluidos $e$, quando \\ apropriado, a descriçăo do processo produtivo. \\ 1.1.1 O(s) propósito(s) e objetivos do desenvolvedor devem ser explicados. \\ LISTA GLASSON, THERIVEL E CHADWICK \\ 1. DESCRICCÄO DO EMPREENDIMENTO \\ 1.1 Principais caracteristicas do projeto \\ 1.1.1 Explica o propósito e objetivo do projeto
}

LISTA COMISSÄO EUROPEIA

1. DESCRICÄO DO PROJETO

1.10 s objetivos $e$ as caracteristicas fisicas do projeto

1.1.1 A necessidade e os objetivos do projeto foram explicados?

\section{LISTA MONTAÑO et al.}

2. CARACTERIZACCÃO DO PROJETO

2.1 Identifica e caracteriza os diferentes componentes do projeto?

2.2 Descreve e explica os objetivos do projeto?

\section{LISTA PINHO, MAIA E MONTERROSO}

\section{CARACTERIZACÃO DO PROJETO}

2.1 Identifica e caracteriza os diferentes componentes do projeto?

2.2 Descreve e explica os objetivos do projeto?

\section{LISTA HICKIE E WADE}

1. OPROJETO

8. Síntese e conclusões - 3 Subtópicos.

9. Relatório de Impacto Ambiental (RIMA) - 6 Subtópicos.

O Tópico 1, "Apresentação de conteúdo", remete, principalmente, a dicas sobre organização do estudo a ser desenvolvido/analisado, como apresentação de um índice, glossário, referências, anexos e a apresentação do conteúdo em si; é uma parte que auxilia na elaboração para que o documento seja mais facilmente entendível e que as informações sejam encontradas de maneira mais rápida, facilitando a análise técnica por parte do órgão ambiental.

O Tópico 2, "Caracterização do projeto", busca fazer com que sejam apresentadas informações a respeito do empreendimento como um todo, o planejamento, a implantação, a operação e, 
quando cabível, a desativação do projeto; assim como conhecimentos sobre gerenciamento de resíduos e emissões e também dos planos emergenciais.

O Tópico 3, "Proposição de alternativas", visa expor todas as alternativas analisadas, tanto locacionais, como tecnológicas e, até mesmo, a alternativa 0 (estudo do estado futuro do ambiente na ausência do empreendimento), bem como apresentar os critérios de escolha da alternativa a ser implementada.

O Tópico 4, "Diagnóstico ambiental”, expõe informações a respeito das condições atuais do ambiente no meio físico, biótico e antrópico antes da implantação do projeto, ou seja, informações sobre o ambiente possivelmente impactado.

O Tópico 5, "Prognóstico ambiental”, é a parte do estudo que analisa os impactos, a partir de métodos específicos, de modo a informar quais serão os possíveis impactos diretos, indiretos, temporários, permanentes, cumulativos, sinérgicos, acidentais e qual "o tamanho" dos mesmos. Aqui também é analisada a significância/importância de cada um destes.

O Tópico 6, "Proposição de medidas ambientais", totalmente dependente dos resultados encontrados no tópico 5 , pois uma vez que se tem uma visão clara dos possíveis impactos significativos gerados pelo empreendimento é necessário elaborar as medidas de minimização, mitigação, compensação e/ou valorização deles.

O Tópico 7, "Programas de monitoramento", depende diretamente dos tópicos 5 e 6 , pois a partir da identificação dos impactos e das propostas de medidas ambientais é preciso formular planos para conferir se estas estão funcionando como o planejado. Para isso, estipulam-se programas de monitoramento que informam qual parâmetro medir, onde medir e/ou quais indicadores devem ser analisados e de quanto em quanto tempo é preciso coletar tais informações.

O Tópico 8, "Síntese e conclusões", apresenta questões que buscam organizar os principais levantamentos do estudo e as conclusões de todo o estudo, apontando a (in)viabilidade ambiental do projeto.

Por fim, o Tópico 9, "RIMA", oferece o detalhamento de como elaborar/analisar este estudo de forma a ser compreensível a leigos, conter as informações relevantes e adequadas de todo o projeto e apresentar confiabilidade.

Para a análise/verificação dos tópicos do roteiro pode-se adotar conceitos propostos por Sanchéz (2008), Lee et al. (1999), European Comission
(2001). No entanto, a proposta deste trabalho é que para cada item seja atribuída uma das 5 classificações de qualidade de avaliação, disposta a seguir:

$$
\begin{aligned}
& 1 \text { - Contempla Completamente (CC) } \\
& 2 \text { - Contempla Incompleto (CI) } \\
& 3 \text { - Somente Cita (SC) } \\
& 4 \text { - Não Contempla (NC) } \\
& 5 \text { - Não se Aplica (NA) }
\end{aligned}
$$

Sendo que "Contempla Completamente" informa que o estudo apresenta a informação de maneira satisfatória, com todos os parâmetros essenciais dispostos e claramente demonstrados, e quando cabível, utilizando de mapas, fotografias, gráficos, etc. "Contempla Incompleto" é uma forma de demonstrar que o estudo não fez uma profunda investigação sobre o assunto quando deveria, possui baixa quantidade de detalhes e poucas figuras demonstrativas. "Somente Cita" faz referência a algo que foi apresentado no estudo de forma superficial, somente citado em meio ao texto de determinado tópico, sem, contudo, explorá-lo. "Não Contempla" é aplicado quando uma informação não é analisada no estudo ou é omitida. E, por fim, "Não se Aplica" é usado quando a questão proposta na lista não se aplica ao empreendimento que está sendo analisado. Nesse último caso, é importante ressaltar a necessidade de discernimento entre os assuntos de maneira crítica, sabendo avaliar quais tópicos seriam indispensáveis para o estudo de determinado empreendimento.

No caso de utilização deste guia para análise do EIA, reafirma-se que uma mera classificação ou atribuição de notas não substitui uma análise crítica e técnica do estudo analisado, apenas auxilia no entendimento e na leitura do mesmo. Sendo assim, como lista de verificação este trabalho pode ser entendido como um instrumento para auxiliar nesta análise técnica e em conjunto com outros instrumentos julgar a qualidade do estudo apresentado e a viabilidade ambiental do empreendimento.

\section{Considerações finais}

O propósito do trabalho foi o de apresentar um roteiro que poderia padronizar e/ou auxiliar a forma de elaboração ou verificação de um EIA, assim, facilitando o desenvolvimento do mesmo por uma equipe técnica responsável ou na avaliação por parte dos tomadores de decisão.

$\mathrm{O}$ roteiro proposto pode ser usado tanto por técnicos de uma consultoria que elaborará o EIA, quanto pelos analistas do órgão ambiental competente pelo processo de licenciamento, no 
momento em que se avalia a qualidade do estudo recebido por parte do empreendedor.

Espera-se com o roteiro diminuir a dificuldade e a subjetividade na elaboração e na avaliação do EIA e contribuir para que estudos de maior qualidade sejam elaborados e, com isso, haja uma melhora na aplicação dos instrumentos Avaliação de Impacto e Licenciamento Ambiental.

\section{Referências}

Almeida, M. R. R., Malfará, D. T., Mendes, N. C., Moraes, M. C. P., Souza, M. P., Montaño, M., 2012. Aplicação e métodos para a revisão da qualidade de impacto ambiental. Revista de Gestão Ambiental e Sustentabilidade 1, 1-28.

Almeida, M. R. R.; Alvarenga, M. I. N.; Cespedes, J. G. 2014. Avaliação da qualidade de estudos ambientais em processos de licenciamento. Revista Geociências, 33, 106-118.

Appiah-opoku, s., 2001. Environmental impact assessment in developing countries: the case of Ghana. Environmental Impact Assessment Review 2, 159-71.

BRASIL, 1981. Lei $\mathrm{n}^{\circ} 6938$ de 31 de agosto.

CONAMA. Conselho Nacional do Meio Ambiente (Brasil), 1986. Resolução $\mathrm{n}^{\circ}$ 001, de 23 de janeiro. Brasília.

Cremonez, F. E., Cremonez, P. A., Feroldi, M., Camargo, M. P., Klajn, F. F., Feiden, A. 2014. Avaliação de impacto ambiental: metodologias aplicadas no Brasil. Revista Monografias Ambientais, 13, 3821-3830.

European Comission, 2001. Guidance on EIA, Eis Review. Disponível: http://ec.europa.eu/environment/eia/review.ht m. Acesso: 1 mai. 2017.

Glasson, J., Therivel, R., Chadwick, A., 1999. Introduction to environmental impact assessment. 2. Ed. London. UCL Press.

Gomes, R. C., 2004. Who are the relevant stakeholders to the local government context? Empirical evidences on environmental influences in the decision-making process of english local authorities. Brazilian Admnistration Review 1, 34-52.

Hickie, D., Wade, M., 1998. Development of guidelines for improving the effectiveness of environmental assessment. Environmental Impact Assessment Review 18, 267-287.

IAIA. International Association for Impact Assessment, 1999. Principles of environmental impact assessment best practice. Fargo: IAIA, Special Publication 1.
Junior, L. S., Alvarenga, M. I.N., Garcia, S. R. 2018. Avaliação da qualidade dos processos de licenciamento ambiental de empreendimentos de mineração em Minas Gerais. Ambiente \& Sociedade, 21, 1-22.

Kruopienė, J., Židonienė, S., Dvarionienė, J., 2009. Current practice and shortcomings of EIA in Lithuania. Impact Assessment and Project Appraisal 29, 305-309.

Leduc, G., Raymond, M.., 2000. L'évaluation des impacts environnementaux: um outil d'aide à ladécision. Ste.-Foy: Multimondes.

Lee, N., Colley, R., Bonde, J., Simpson, J., 1999. Reviewing the quality of environmental statements and environmental appraisals. University of Manchester. Manchester.

Leite, M.M. 2013. Análise comparativa dos sistemas de avaliação de impacto ambiental. In: LIRA, WS., and CÂNDIDO, GA., orgs. Gestão sustentável dos recursos naturais: uma abordagem participativa [online] 273-293. ISBN 9788578792824. Available from SciELO Books . Acesso 23 jul. 2018.

Lima, L. H., Magrini, A. 2010. The Brazilian Audit Tribunal's role in improving the federal environmental licensing process. Environmental Impact Assessment Review 30, 108-115.

Mendes, D., Feitosa, A., 2007. IBAMA reduzirá em mais de $50 \%$ prazo para concessão de licença ambiental. Disponível: www.mma.gov.br/ascom/ultimas/index.cfm?id $=4241$. Acesso 10 jan. 2017.

Montaño, M., Carvalho, A. F., Gomes, C. S.; Polaz, C. N. M., Jordão, C. O., Souza, M. P., 2014. Revisão da qualidade de estudos de impacto ambiental de pequenas centrais hidrelétricas. Holos Environment 14, 1-14.

Morgan, R. K. 2012. Environmental impact assessment: the state of the art. Impact Assessment and Project Appraisal, 1, 5-14.

MPF - Ministério Público Federal, 2004. Deficiências em Estudos de Impacto Ambiental: síntese de uma experiência. Brasília: $4^{a}$ Câmara de Coordenação e Revisão, Escola Superior do Ministério Público da União.

Panigrahi, J. K., Amirapu, S., 2012. An assessment of EIA system in India. Environmental Impact Assessment Review 35, 23-36.

Pinho, P., Maia, R., Monterroso, A., 2007. The quality of Portuguese environmental impact studies: the case of small hydropower projects. Environmental Impact Assessment Review 27, 189-205. 
Pope, J., Bond, A., Morrison-Saunders, A., Retief, F. 2013. Advancing the theory and practice of impact assessment: Setting the research agenda. Environmental Impact Assessment Review 41, 1-9.

Prado Filho, J. F., Souza, M. P., 2004. O Licenciamento Ambiental da mineração no Quadrilátero Ferrífero de Minas Gerais - uma análise da implementação de medidas de controle ambiental formuladas em EIAS/RIMAS. Engenharia Sanitária e Ambiental 9, 343-349.

Ross, W. A., Morrison-Saunders, A., Marshall, R., 2006. Common sense in environmental impact assessment: it is not as common as it should be. Impact Assessment and Project Appraisal 24, 3-22.

Sánchez, L. E., 2008. Avaliação de impacto ambiental: conceitos e métodos. Oficina de Textos, São Paulo.

Sánchez, L. E., 2013. Avaliação de impacto ambiental: conceitos e métodos. $2^{\mathrm{a}}$ ed.: Oficina de Textos, São Paulo.

Sandoval, M., Cerri, L., 2009. Proposta de padronização em avaliação de impactos ambientais. Engenharia Ambiental - Espírito Santo do Pinhal, 6, 100 - 113.

Santiago, C. S.; Alvarenga, M. I. N.; Almeida, M. R. R. 2016. Avaliação da etapa de acompanhamento do licenciamento ambiental de abatedouros e laticínios em Minas Gerais. Revista Brasileira de Geografia Física, 9, 940954.

Tommasi, L. R., 1994. Estudo de impacto ambiental. Cetesb/ Terragraph, São Paulo.

Turra, A., Amaral, A. C. Z., Ciotti, A. M., Wongtschowski, C. L.D.B.R., Novelli, Y. S., Marques, A. C., Siegle, E., Sinisgalli, P. A. A., Santos, C. R., Carmo, A.B. 2017. Avaliação de impacto ambiental sob uma abordagem ecossistêmica: ampliação do porto de São Sebastião. Ambiente \& Sociedade, 20, 159-178.

Tzoumis, K., 2007. Comparing the quality of draft environmental impact statements by agencies in the United States since 1998 to 2004. Environmental Impact Assessment Review 27, 26-40.

Vanclay, F. 2015. Changes in the impact assessment family 2003-2014: implications for considering achievements, gaps and future directions. Journal of Environmental Assessment Policy and Management, 17, 20.

Wärnbäck, A., Hilding-Rydevika, T., 2009. Cumulative effects in Swedish EIA practice: difficulties and obstacles. Environmental Impact Assessment Review. 29, 107-115.

Zubair, L., 2001. Challenges for environmental impact assessment in Sri Lanka. Environmental Impact Assessment Review 21, 469-478.

\section{APÊNDICE I}

\section{Lista de Verificação/Roteiro Geral}

Este apêndice refere-se a um roteiro de elaboração ou um guia de análise de um Estudo de Impacto Ambiental (EIA) para ser aplicado a várias tipologias de empreendimentos. Para cada tipo de projeto é necessário analisar quais as questões fundamentais são necessárias no estudo. O roteiro tem como base uma estrutura típica de um EIA, sendo que cada tópico ou subtópico busca trazer questões específicas que devem ser discutidas em um estudo completo e de boa qualidade.

\section{Apresentação de conteúdo \\ 1.1Sumário/índice}

1.1.1 Apresentar um sumário paginado que permita encontrar rapidamente um assunto de interesse.

\subsection{Glossário de termos técnicos}

1.2.1 Explicar detalhadamente termos técnicos e siglas em glossário ou em texto.

\subsection{Referências}

1.3.1 Referenciar devidamente todas as fontes externas de dados, informações, abordagens, citações bibliográficas e estudos não publicados, com indicação dos locais onde podem ser consultados.

\subsection{Anexos/Apêndices}

1.4.1 Fazer uso de anexos e apêndices para mostrar dados específicos, mas não essenciais para entendimento do texto no geral, na apresentação das informações.

1.4.2 Ter atenção para que nenhum anexo citado no texto deixe de ser apresentado e nenhum anexo apresentado deixe de ser chamado no texto.

\subsection{Equipe técnica}

1.5.1 Apresentar a empresa de consultoria que elaborou o estudo.

1.5.2 Identificar os participantes do estudo com seus respectivos nomes, números de 
registro profissional e qualificações. Além de mencionar a contribuição de cada um no estudo.

\subsection{Apresentação do documento}

1.6.1 Elaborar um estudo claro, sem informações irrelevantes e bem escrito.

1.6.2 Organizar lógica e claramente os documentos para que o leitor possa achar as informações facilmente.

1.6.3 Usar de tabelas, imagens, mapas, fotografias e outros recursos gráficos para melhor entendimento do estudo.

1.6.4 Reconhecer e explicar os meios usados para lidar com qualquer dificuldade na montagem ou análise dos dados do estudo e também fornecer qualquer base para questionar pressupostos, dados ou informações.

1.6.5 Informar qual legislação foi utilizada como base no estudo e demonstrar que a atendeu adequadamente.

1.6.6 A não ser que os capítulos sejam bem curtos, recomenda-se haver um resumo dos principais achados em cada fase da investigação.

\section{Caracterização do projeto}

\subsection{Empreendimento}

2.1.1 Especificar nome do responsáveis e endereço de contato.

2.1.2 Explicar o propósito e objetivo do projeto.

2.1.3 Expor clara e precisamente as fontes de recursos financeiros.

2.1.4 Descrever o local, concepção e tamanho do empreendimento. Para isso, utilizar do auxílio de diagramas, planos, mapas e/ou fotografias.

2.1.5 Descrever o layout do local ocupado) pelo projeto. (Incluir os níveis do solo, edifícios, outras estruturas físicas, obras subterrâneas, obras costeiras, instalações de armazenamento, recursos hídricos, plantação, corredores de acesso, limites).

\subsection{Fase de Planejamento}

2.2.1 Descrever a relação entre o projeto em âmbito local, regional e nacional. Diagramas, mapas e planos podem ser utilizados com este objetivo.

2.2.2 Descrever novas infraestruturas de transportes que influenciarão substancialmente fluxo do tráfego, o tipo, volume, padrão temporal e distribuição geográfica do novo tráfego gerado ou alterado, como consequência do projeto.
2.2.3 Caso haja, listar os serviços adicionais que são requeridos pelo projeto. (Por exemplo: acesso de meios de transporte, água, sistema de esgoto, eliminação de resíduos, eletricidade, telecomunicações, estradas, portos, linhas de alta tensão, oleodutos).

2.2.4 Caso haja, listar empreendimentos que possam ocorrer como conseqüência do projeto identificado. (Por exemplo, novas habitações, estradas, infra-estruturas de água ou esgotos, extração de agregados).

2.2.5 Caso haja, listar a existência de atividades que possam alterar ou cessar em consequência do projeto identificado.

2.2.6 Caso haja, listar outros empreendimentos reais ou planejados com os quais o projeto poderia ter efeitos cumulativos identificados.

\subsection{Fase de Implantação}

2.3.1 Descrever todas as atividades envolvidas na construção do projeto.

2.3.2 Listar os tipos, a natureza e quantidades de matérias-primas e as energias utilizadas e produzidas na construção.

2.3.3 Estimar o número de trabalhadores da construtora e de terceiros no local durante a construção do projeto. Fornecer também seus acessos e os meios de transporte para o local.

2.3.4 Discutir a hospedagem e provisão de serviços para funcionários temporários ou permanentes do projeto. (Relevante para projetos que requerem migração substancial de trabalhadores para a área de construção ou de longo prazo).

2.3.5 Apresentar critérios de contratação e dispensa de trabalhadores durante a construção.

2.3.6 Descrever os meios de transporte das matérias-primas e produtos para o local e suas respectivas quantidades aproximadas envolvidas na fase de construção do projeto. 2.3.7 Caracterizar a localização e características de canteiros de obras.

2.3.8 Possuir um cronograma da fase de construção do projeto (estimar a duração desta fase).

\subsection{Fase de Operação}

2.4.1 Descrever todas as atividades e processos envolvidos na operação do projeto.

2.4.2 Listar os tipos, a natureza e quantidades de matérias-primas, energias utilizadas e produzidas na operação. 
2.4.3 Caracterizar, quando apropriado, a natureza do processo de produção que será empregado na empresa e também a expectativa da taxa de produção.

2.4.4 Estimar o número de trabalhadores e de terceiros no local durante a operação do projeto. Fornecer também seus acessos e os meios de transporte para o local.

2.4.5 Apresentar critérios de contratação e dispensa de trabalhadores durante a operação.

2.4.6 Descrever os meios de transporte das matérias-primas e produtos para o local e suas respectivas quantidades aproximadas envolvidas na fase de operação do projeto.

2.4.7 Possuir um cronograma consistente da fase de operação do projeto (estimar a duração desta fase).

\subsection{Fase de Desativação (quando aplicável ao empreendimento)}

2.5.1 Descrever todas as atividades e processos envolvidos na desativação do projeto.

2.5.2 Listar os tipos, a natureza e quantidades de matérias-primas, energias utilizadas e produzidas na desativação.

2.5.3 Possuir um cronograma consistente da fase de desativação do projeto (estimar a duração desta fase).

\subsection{Resíduos, efluentes e emissões}

Válido para construção, operação e

desativação. Resíduos incluem todos os materiais residuais dos processos, efluentes e emissões. Resíduo energético, calor, ruído, etc, também devem ser considerados.

2.6.1 Identificar os tipos e quantidades de resíduos sólido gerado pelo projeto.

2.6.2 Discutir a composição, toxidade ou outros perigos dos resíduos sólidos produzidos pelo projeto.

2.6.3 Descrever os métodos para coleta, estoque, tratamento, transporte e disposição final desses resíduos sólidos.

2.6.4 Discutir o potencial de recuperação dos resíduos. (Incluindo reuso, reciclagem ou recuperação de energia de resíduos sólidos e efluentes líquidos).

2.6.5 Indicar os métodos pelos quais as quantidades de resíduos são obtidos. Se houver incerteza ela deverá ser reconhecida e possíveis limites de confiança fornecidos, sempre que possível.

2.6.6 Identificar os tipos e quantidades de efluentes líquidos gerados pelo projeto.
2.6.7 Discutir a composição, a toxidade ou outros perigos de todos os efluentes líquidos produzidos pelo projeto.

2.6.8 Descrever os métodos de coleta, estoque, tratamento, transporte e disposição final desses efluentes líquidos.

2.6.9 Identificar os tipos de quantidades de emissão de gases e particulados gerados pelo projeto. (Incluindo emissão do processo, as emissões fugitivas, as emissões resultantes da combustão de combustíveis fósseis em instalações estacionárias e móveis, as emissões provenientes do tráfego, as poeiras provenientes da manipulação de materiais, os odores).

2.6.10Descrever a composição, toxidade ou outros perigos de todas as emissões para o ar produzidas pelo projeto.

2.6.11Descrever os métodos de coleta, tratamento e descarregamento final dessas emissões para o ar.

2.6.12Caso haja, caracterizar e quantificar fontes de ruído, calor, luz ou radiação eletromagnética do projeto. (Incluindo equipamento, processos, trabalhos de construção, tráfego, iluminação, etc).

\subsection{Análise de riscos, perigos e acidentes}

Válido para construção, operação e desativação.

2.7.1 Realizar uma avaliação da probabilidade e prováveis consequências de acidentes que possam causar severos danos ao ambiente, se a natureza do projeto é passível disto e reportar os principais achados. (Exemplos: risco na manipulação de materiais perigosos, de fogo ou explosões, de acidentes de tráfego, de ruptura ou falha de processos ou instalações, de exposição do projeto a desastres naturais - tremores, inundação, deslizamentos, etc).

2.7.2 Descrever medidas para prevenção e resposta a acidentes e eventos anormais. (Exemplos: Medidas preventivas, treinamento, planos de contingência e emergência, etc).

\section{Proposição de alternativas}

3.1Elaborar as alternativas de modo que elas sejam realistas e genuínas.

3.2Descrever as várias alternativas em relação à localização do projeto disponíveis.

3.3Descrever as várias alternativas em relação aos processos tecnológicos disponíveis.

3.4Verificar o futuro do estado do ambiente, na ausência do projeto, levando em consideração 
flutuações naturais e atividades humanas (geralmente chamada de alternativa zero).

3.5Considerar, caso tenha ocorrido, as considerações apresentadas em reuniões e consultas públicas.

3.6Comparar as alternativas de forma clara e objetiva e compará-las com as prováveis condições ambientais futuras sem o projeto.

3.7Explicar as razões pelas quais algumas alternativas foram descartadas do estudo.

3.8Descartar alternativas que apresentem algum critério de inviabilidade.

3.9Fornecer uma indicação das principais razões para a alternativa escolhida, os critérios utilizados para a escolha, levando em consideração as principais vantagens e desvantagens dos efeitos ambientais disso.

\section{Diagnóstico ambiental}

\subsection{Orientações gerais}

4.1.1 Definir o ambiente afetado de modo a incluir qualquer efeito potencial significante que ocorram longe das áreas imediatas de construção e operação (exemplo: pela dispersão de poluentes, requisitos de infraestrutura do projeto, tráfego).

4.1.2 Referenciar, adequadamente, as fontes de dados e informações do ambiente existentes. Explicar caso alguma importante lacuna nos dados do ambiente for identificada, como também explicar os meios utilizados para lidar com ela durante a avaliação.

4.1.3 Identificar e descrever os componentes importantes do ambiente afetado. Divulgar os métodos e investigações realizados para esse fim, sendo apropriados ao tamanho e complexidade dessa tarefa de avaliação.

4.1.4 Atender, obrigatoriamente, o Termo de Referência apresentando todas as informações requeridas sobre o meio físico, biótico e antrópico.

4.1.5 Elaborar mapas e representações dos processos e/ou fenômenos do meio físico, biótico e antrópico com escalas adequadas.

4.1.6 Focar nas informações relevantes, descartando informações supérfluas ou irrelevantes para a tomada de decisões.

4.1.7 Descrever, com adequado detalhe, os métodos utilizados para os diferentes levantamentos.

\subsection{Físico}

4.2.1 Expor características significantes do relevo e geomorfologia da área descrita e as condições, tipos e uso dos solos. (Isso inclui estabilidade e resistência à erosão do solo, uso para outras atividades e qualidade da terra agrícola).

4.2.2 Identificar, adequadamente, áreas com potenciais problemas de ordem geotécnica.

4.2.3 Descrever a água no ambiente do projeto. (Incluindo águas superficiais lóticas e lênticas, águas subterrâneas, estuários, resíduos litorais e do mar)

4.2.4 Relatar a hidrologia, qualidade da água e uso de qualquer recurso aquático que pode ser afetado pelo projeto. (Incluindo uso para abastecimento, pesca, banho, amenidade, navegação, disposição de efluentes).

4.2.5 Mapear os principais usos da água a jusante da obra.

4.2.6 Caracterizar as condições climáticas e meteorológicas e a qualidade existente do ar na área do projeto.

4.2.7 Especificar a paisagem da área que pode ser afetada pelo projeto.

4.2.8 Identificar e mapear zonas com problemas críticos de ruído.

\subsection{Biótico}

4.3.1 Caracterizar e ilustrar em mapas apropriados a fauna, a flora e habitats da área a ser ocupada pelo projeto e seu entorno.

4.3.2 Descrever espécies de populações e características dos habitats que possam ser afetados pelo projeto.

4.3.3 Conferir se alguma espécie é protegida nas áreas definidas.

4.3.4 Identificar e mapear, obrigatoriamente, ocorrências de vegetação nativa, principalmente de biomas comprometidos (Hot Spots, alguns exemplos são: Cerrado, Mangues, Mata atlântica, etc).

4.3.5 Identificar as ares protegidas que podem ser impactadas pelo projeto.

\subsection{Antrópico}

4.4.1 Caracterizar a área de estudo quanto a aspectos de dinâmica da população, fatores culturais e dados sociais e econômicos.

4.4.2 Descrever os usos da terra existentes na área a ser ocupada pelo projeto e seu entorno. Conferir se existem pessoas que vivem ou usam essas terras. (Incluindo usos residenciais, comerciais, industriais, agrícolas, recreativos e de amenidades, edifícios, estruturas ou outros bens).

4.4.3 Verificar se há algum local ou característica arqueológica, histórica, arquitetônica, outra comunidade ou 
importância cultural na área que pode ser dividida pelo projeto descrito. (Incluindo qualquer local protegido).

4.4.4 Se houver, caracterizar as populações a serem deslocadas.

\section{Prognóstico ambiental (impactos) Identificação e previsão}

5.1 Identificar e selecionar impactos chave para uma investigação mais intensa.

5.2Descrever os efeitos diretos sobre os principais componentes do meio físico, biótico

e antrópico nas diferentes etapas do empreendimento (planejamento, construção, operação e desativação).

5.3 Apresentar a metodologia para a identificação e a predição de impacto e indicar a incerteza associada a cada predição. Explicar os métodos utilizados e fornecer as razões para a escolha do nível de detalhe da análise. (Exemplos: listas de verificação específicas, matrizes, painéis de especialistas, consultas).

5.4Expressar, em quantidades mensuráveis, previsões de impacto com taxas e/ou limites de confiança apropriados. Definir descrições qualitativas, onde são usadas, tão completamente quanto possível (Exemplo: meios insignificantes não perceptíveis a partir de mais de $100 \mathrm{~m}$ de distância).

5.5 Aplicar métodos que sejam apropriados à extensão geográfica, duração, frequência, reversibilidade e probabilidade de ocorrência de cada efeito identificado.

5.6 Descrever efeitos indiretos no ambiente causados como desenvolvimento consequencial e, quando possível, quantificá-los.

5.7Expor os efeitos que podem ser resultado de acidentes, eventos anormais ou exposição do projeto a catástrofes naturais ou provocadas pelo homem e, quando possível, quantificá-los 5.8Descrever efeitos no ambiente causados por atividades auxiliares do projeto principal e, quando possível, quantificá-los.

5.9Especificar efeitos cumulativos e sinérgicos no ambiente fora do projeto juntamente com outros empreendimentos existentes ou previstos na localidade e, quando possível, quantificá-los.

\section{Avaliação da Significância}

5.10 Classificar os impactos positivos e negativos (benéficos e adversos); diretos e indiretos; imediatos e a médio e longo prazos; temporários e permanentes; seu grau de reversibilidade; suas propriedades cumulativas e sinérgicas; a distribuição dos ônus e benefícios sociais.
5.11 Incluir clara indicação de quais impactos podem ser significantes e quais não, e fornecer justificativas para tais distinções.

5.12 Discutir a significância ou importância de cada efeito previsto em termos de seu cumprimento legal e o número, importância e sensibilidade das pessoas, recursos ou outros receptores afetados.

5.13 Avaliar a significância de um impacto levando em consideração padrões de qualidade nacionais e internacionais apropriados, onde disponível.

5.14 Considerar impactos que podem não ser tão significantes quando analisados isoladamente, mas que podem contribuir substancialmente para um efeito cumulativo significante.

5.15 Justificar a escolha dos padrões, suposições e sistema de valores usados para avaliar a significância e resumir qualquer opinião contrária. Discutir a significância dos efeitos em termos de impactos na comunidade local (incluindo distribuição dos impactos) e na proteção dos recursos naturais.

5.16 Evitar julgamentos subjetivos de valor.

\section{Proposição de medidas ambientais}

6.1Obedecer a escala da medida de mitigação: evitar, reduzir, compensar e recuperar.

6.2Fornecer uma descrição das medidas previstas, sendo estas compatíveis com os impactos causados e tendo seus efeitos na magnitude e significância do impacto claramente explicados.

6.3Propor medidas que permitam valorizar os impactos positivos.

6.4 Detalhar como as medidas de mitigação serão implantadas e como funcionarão ao longo do tempo para o qual são necessárias. Descrever as razões para escolha particular de cada tipo de mitigação e as outras opções disponíveis. (Exemplos: compensação pela perda de habitats mediante proteção de área equivalente e/ou recuperação de áreas degradadas).

6.5 Deixar claro qual a extensão da implementação e da efetividade do método de mitigação/minimização/compensação quando este for implementado.

6.6Definir claramente as responsabilidades para implementação da mitigação e/ou compensação.

6.7Justificar, obrigatoriamente, as razões quando a mitigação de impactos adversos significativos não for praticável ou o empreendedor optou por não propor nenhuma mitigação. 
6.8Descrever alguns possíveis efeitos negativos na proposta de mitigação, levando em consideração um potencial conflito entre os benefícios das medidas de mitigação e seus impactos adversos.

\section{Programas de monitoramento}

7.1Propor o acompanhamento para todos os impactos significantes, especialmente aqueles com incerteza, para avaliar o ambiente impactado e sua conformidade com as previsões feitas.

7.2Descrever os objetivos do programa de monitoramento e sua justificativa científica de todos os fatores ambientais.

7.3Descrever com precisão quais tipos de indicadores devem ser monitorados, como e quando devem ser monitorados e que organização/instituição deve ser responsável pela fiscalização.

7.4Fazer com que a escala de qualquer disposição de acompanhamento proposta corresponda a potencial escala e significância de desvios esperados dos impactos.

7.5Fornecer evidências do compromisso dos proponentes com $\mathrm{o}(\mathrm{s})$ programa(s) de monitoramento.

7.6Propor adequadamente as localizações das estações e as frequências de amostragem e informar os métodos de coleta e análise de dados.

\section{Síntese e conclusões}

8.1Apoiar todas as análises e conclusões em dados e evidências.

8.2Retomar as principais conclusões do EIA nessa seção.

8.3Apresentar as conclusões finais sobre os impactos positivos e negativos da proposta, considerando as diferentes fases do projeto e apontando a sua (in)viabilidade ambiental.

\section{Relatório de impacto ambiental (RIMA)}

9.1Evitar termos técnicos, lista de dados e explicações detalhadas ou raciocínios científicos. Evitar o que seria de difícil compreensão para um público leigo.

9.2Elaborar o RIMA de modo que ele seja suficientemente conciso para permitir uma leitura rápida e com linguagem adequada.

9.3Fornecer uma breve descrição do projeto, do ambiente, dos efeitos do projeto no ambiente, suas alternativas, propostas de mitigação/compensação, monitoramento e descrição de quaisquer impactos residuais.

9.4Apresentar os principais achados e conclusões da avaliação realizada.

9.5Indicar confiabilidade, na qual pode ser conferida nos resultados.

9.6Expor ilustrações que expliquem satisfatoriamente o projeto. 